\title{
A physical psychological factor in immunostimulation activity and homeostasis safeguarding
}

\author{
Nafissa TELAILIA ${ }^{1}$ and Hacène FRIH ${ }^{1}$ \\ ${ }^{1}$ Université Badji Mokhtar Annaba Faculté des Sciences
}

May 5, 2020

\begin{abstract}
Abstract The objective of our study is to propose a therapeutic strategy based on the administration of cyclosporine alone, and cyclosporine associated with a sound noise in two batches of animals treated with an immunostimulant Biliated bacillus of Calmette and Guerin, all based on the hypothesis of the central role of neuroimmuno-endocrine regulation loop in disorders related to the administration of cyclosporine. We administered cyclosporine alone (IP) for 8 days, as well as administration of cyclosporine associated with sound noise with food and sugar water and stained in two batches of Biliated bacillus of Calmette and Guerin -treated animals ( in ID), the animals received Biliated bacillus of Calmette and Guerin injections on the 9th day and a reminder on the $27 \mathrm{th}$ day. Our results show that intraperitoneal injection of cyclosporine alone $(20 \mathrm{mg} / \mathrm{kg})$ causes a significant decrease in testosteronemia associated with a drop in body weight. Similar results were found following administration of cyclosporine associated with sound noise. Conversely, administration of cyclosporine alone and associated with sound noise caused a significant increase in adrenocorticotropic hormone. Indicating that sound noise mimics the effect of cyclosporine in male rats. Keywords: Immunosuppression, immunostimulation, psychotherapy, homeostasis
\end{abstract}

\section{Introduction}

Controlling the immune system response is a major challenge in human medicine. Modifiers of the immune response (Labro et al., 2006)are called immunomodulators. Depending on their pharmacological action on the immune system, these agents can stimulate or suppress the immune system. Interest in the subject of immunomodulation has grown rapidly in recent years, with the realization of its potential in human medicine in the fields of cancer therapy, the treatment of autoimmune diseases and transplant technology. Several recent studies bear witness to this. However, therapeutic applications of the immune modulator, drugs are still limited by our incomplete knowledge of how their effects are produced (Fauve et al., 1980) . The term "immunomodulation" is generally used to describe the pharmacological manipulation of the state of activity of the immune system (Skarbek et al., 2017). This may involve an increase in the magnitude of immunostimulation immune responses or a decrease in the magnitude of immunosuppression. Specific immunomodulation involves a change in the response of the system to a particular antigenic stimulus (Genard et al., 2015) , as caused by the vaccination (specific immunostimulation) and desensitization (specific immunosuppression) processes. Specific immunomodulation involves a more fundamental change by which the state of alertness of the immune system is altered, which affects the nature of its responses to the multiplicity of antigenic stimuli. The most commonly used type of immunomodulation in medicine is immunosuppression; which is often accomplished by the use of immunosuppressive molecules (azathioprine, cyclosporine, danazol, etc.) (Hill et al., 2017) . The discovery in 1972 by JF Borel of the immunosuppressive activity of ciclosporin A a cyclic peptide of 11 amino acids (C6H11N11O12), extracted from a fungus discovered in 1969, Tolypocladium inflatum, and its use for the first time in human clinic in allograft kidney and bone marrow recipients. Although it was initially identified as an antibiotic agent, it was later discovered to be a potent immunosuppressive agent (Borel et al., 1976) . It is successfully used in transplant medicine and 
in the treatment of autoimmune diseases such as uveitis(Nussenblatt et al., 1987), rheumatic arthritis(Yoshinoya et al., 1988) and psoriasis (Mihatsch et al., 1992). Cyclosporine A has been used successfully in several immunologically based diseases and in transplant patients. However, therapeutic treatment induces several side effects, including the development of severe hypertension, renal failure and cardiotoxicity in the majority of patients. Because the mechanism by which cyclosporine induces hypertension is not well defined. In addition, recent studies have shown that cyclosporine plays a neuroprotective role(Friberg et al., 1998), as well as trophic effects(Borlongan et al., 1999) and modifies behavioral activity in normal animals and models. Humans who have diseases of the neurodegenerative disorder (Tomac et al., 1999) . Thus, cyclosporine is both a powerful tool for elucidating neurotrophic mechanisms and a potential therapeutic agent in brain disorders, such as Alzheimer's disease (Borlongan et al., 2000) . Clinical immunosuppressive treatments using cyclosporine induce changes in the mental state of patients (Mason et al., 1990) in 20\% of transplants of kidney (Kahan et al., 1987) and liver(De Groen et al. ., 1987). Therefore studies in rodents are necessary to study the affective disorders induced by Cyclosporine. Studies in animals suggest that cyclosporine inhibits the development of cell-mediated reactions, it blocks lymphocyte activity without modifying the DNA of lymphocytes and ensures a rapidly reversible action. It acts selectively on $\mathrm{T}$ cells with the CD4 phenotype, responsible for the initial recognition of the antigen and for the secretion of pro-inflammatory cytokines, in particular interleukin 2 (IL-2) or the T cell growth factor (TCGF). ). It quickly revealed an immunosuppressive activity superior to that of zanzathioprine (Cohen et al., 1984) .Immunostimulants are agents that trigger the specific immune response and lead to improved disease resistance (Gerbase et al., 2000). Several compounds are said to have immunostimulatory properties. Immunostimulation uses various chemicals, including adjuvants, to enhance natural or artificial immunization (via vaccination) with the use of bacterial extracts, B.C.G or corynebacteria, is a vaccine against tuberculosis (TB). Biliated bacillus of Calmette and Guerin is used in many countries with a high prevalence of tuberculosis to prevent tuberculous meningitis and miliary disease in children (Billy et al., 2004) . However, Biliated bacillus of Calmette and Guerin is generally not recommended in the United States due to the low risk of Mycobacterium tuberculosis infection, the variable efficacy of the vaccine for adult pulmonary tuberculosis and the potential interference of the vaccine with skin reactivity. tuberculin. The Biliated bacillus of Calmette and Guerin vaccine should only be considered for highly selective people meeting specific criteria and in consultation with a tuberculosis expert. The efficacy of the Biliated bacillus of Calmette and Guerin vaccine has been a recurrent subject due to divergent test results (C.R. Macintyre, New developmentin BCG vaccine: implicatuin for tuberculosis control, Epidemiol Infect. 2007 Febrary). The goal of immunostimulation is to increase the effectiveness of the immune system against a pathogen. The advantage of Biliated bacillus of Calmette and Guerin injections is to boost immunity. To promote the recognition of pathogenic cells and their destruction by the immune system. The idea that the immune system can be modulated in its functioning by the brain came from theories of stress. Recent developments in psychoneuroimmunology which aims to study the interactions between the nervous system and the immune system have revealed the existence of a whole network of communications between these two systems. Lymphoid organs are innervated by the autonomic nervous system (Dantzer et al., 2003) - Immune system cells have receptors on their surface for conventional neurotransmitters and neuropeptides (Stora et al., 2016) . Activation of these receptors results in functional changes in immune cells, in the form of changes in the proliferation, chemotaxis, differentiation or immune function of the cells under consideration. Given this circuitry, it is not surprising that brain damage or exposure to aggression results in impaired immune responses. These alterations in fact represent the counterpart of the regulation of the immune system by the brain. The nervous system is effectively able to follow the activity of the immune system, via the inflammatory cytokines released by the cells of innate immunity and acting on the brain via the afferent nerves(Ader et al., 1990). The immune patterns of pathogens are also capable of acting on the brain, in the circumventricular organs. In both cases, the peripheral immune signal is relayed by the synthesis and release of inflammatory cytokines in the brain. These diffuse through the brain parenchyma and, acting on their neural targets, organize the central component of the host response to infection, marked by fever, neuroendocrine activation and the development of disease behavior(Kelley et al., 2017) . Pavlov's reflex (Jarius et al., 2017) is a conditional reflex highlighted by Ivan Pitrovitch, Pavlov who gave it his 
name. Pavlovian conditioning is often said, from 1889, the physiologist showed that if one accustomed a dog to accompany its food with a sound stimulus, the latter could in the long term trigger the salivation of the animal without being accompanied by food. In fact, it has been shown that the secretion of saliva can be caused by direct contact with food or by a stimulus linked to it, such as a bell sound. Pavlov has made considerable progress in researching conditional reflexes, which can be likened to an unborn innate reaction, caused by an external stimulus. Pavlov developed the theory that reactions acquired through learning and habit become reflexes when the brain makes the connections between the stimulus and the action that follows. The most elaborate example of the immune system's sensitivity to psychological factors is the conditioning of immune responses, usually using the administration of an immunomodulatory treatment, cyclosporine for example, as an unconditional stimulus (Bockaert et al., 2017) and l ingestion of a food with a particular taste, for example diluted saccharin, as a conditional stimulus (Grastyan et al., 1966) . After a sufficient number of combinations, the presentation of the conditional stimulus alone is supposed to evoke the image of immunosuppressive therapy and thus accentuate immunosuppression. Numerous results obtained in different contexts, varying by the type of immune response studied, the nature of the conditional stimulus and that of the unconditioned stimulus, confirm the occurrence of relative immunosuppression in conditioned animals but not in control animals. Given the modalities of the tests, it is not always easy to differentiate a real conditioning of the immune response from effects due to stress and the discomfort felt by animals during re-exposure to a signal associated with the initial state of malaise, caused by the immune stimulus studied. Our experiment aims to study the combined effect of an immunosuppressant (intra-penitential injection of cyclosporine) and an immunostimulator (intradermal Biliated bacillus of Calmette and Guerin injection), conditioned to stimulation with sound noise in male Westar rats.

\section{Materials and methods}

\section{Biological materials}

In this study, we used male rats Ratus of the Wistar strain, 6 months old (from the Pasteur Institute in Algiers), weighing between 190 and $250 \mathrm{~g}$. The rats were housed in polypropylene cages. The litter made of wood chips is changed every other day. The ambient conditions of breeding are well controlled; the photoperiod is 12 "h" of light and 12 "h" of darkness (light on at 07:00 am) and the temperature is $22 \pm$ $2^{\circ} \mathrm{C}$. Animals had ad libitum access to food and water. Animals received food in the form of sticks made of corn, bran, soy, barley, milk and vitamin supplements provided by the National Office of Livestock feed (Ets ONAB, El Kseur- Béjaïa) and tap water in bottles. After an adaptation period of 1 month, 21 male rats were divided into 3 groups of seven rats:

-Cyclosporine group: injection of ciclosporin at $0.02 \mathrm{ml} / \mathrm{kg}$ intraperitoneally (IP) for a period of 8 consecutive days

\section{(Kamilia, Hacène et al. 2015)}

- Control groups: the control animals underwent an injection of equivalent volume $0.02 \mathrm{ml} / \mathrm{kg}$ in (PI) $0.9 \%$ saline solution.

-Group Noise + cyclosporine: consists in applying a noise of an intensity of $105 \mathrm{db}$ for 2 min associated with an injection of a dose of $0.02 \mathrm{ml} / \mathrm{kg}$ of cyclosporine intraperitoneally during eight consecutive days.

\section{Treatment of animals}

\section{Sound noise device}

The sound noise device is represented by a polyethylene box (L. 38.5cmx W.24cmx H. 16cm) and a perforated cover for ventilation which allows the animal to be brought in and locked in. Inside the box, a model horn $(12 \mathrm{~V}, 3 \mathrm{~A}, 105 \mathrm{db}, 510 \mathrm{KHZ})$ is attached and held on one side of the box. The horn is connected to an anlium type adapter $(12 \mathrm{~V}, 5 \mathrm{~A})$ to provide AC power. The stress test involves placing the animal in the device and starting the horn for 2 minutes.

\section{Administration of an immunosuppressant "cyclosporine A"}


Cyclosporine is an immunosuppressive agent whose therapeutic use, from the beginning of the 1980s, allowed a considerable development in the field of organ transplantation by preventing the acute rejection of allografts. It is a cyclic peptide of eleven amino acids synthesized by a microscopic fungus, Tolypocladiuminflatum. Cyclosporine A has immunregulatory properties by inhibiting the activation of both $\mathrm{B}$ and $\mathrm{T}$ lymphocytes (Hess et al., 1982). In our study, cyclosporine was injected intraperitoneally at a dose of $20 \mathrm{mg} / \mathrm{kg}$ (Guedri et al., 2014) . Cyclosporine injections were given for 8 days because of one injection / day. This test was carried out using the Biomerieux MINI VIDAS device (100 $\mu$ l plasma serum).

Remarque: On the 28th day, the application of noise is resumed for the Noise + Cyclosporine group without injection of cyclosporine for four days before the blood sample. On the $28^{\text {th }}$ day, the cyclosporine batch receives cyclosporine for four days.

\section{Administration of an immunostimulator "B.C.G" Bacillus Calmette and Guérin (Mycobacte- riumbovis)".}

Biliated bacillus of Calmette and Guerin is a living strain of Mycobacteriumbovis, attenuated by successive passages on culture media. Its initial use was medical prophylaxis for tuberculosis. The lyophilized Biliated bacillus of Calmette and Guerin vaccine comes from the culture of the Connaught sub-strain of bacillus Calmette Guérin, an attenuated strain of Mycobacteriumbovis Karlson and Lessel, 1970 suspended in monosodium glutamate. Biliated bacillus of Calmette and Guerin cultures are spread on a Sauton growth medium, then mixed with lyophilized monosodium glutamate. Biliated bacillus of Calmette and Guerin cultures are viable after reconstitution. The Biliated bacillus of Calmette and Guerin vaccine is supplied in multi-dose vials accompanied by a diluent composed of a sterile saline solution buffered with phosphate containing $0.025 \%$ of polysorbate 80 . The concentration of the reconstituted vaccine is from $8 \times 105$ to $32 \times 105$ units forming colonies (CFU) per adult dose of $0.1 \mathrm{~mL}(=0.1 \mathrm{mg}$ Biliated bacillus of Calmette and Guerin), equivalent to $4 \times 105$ to $16 \times 105 \mathrm{CFU}$ per child dose of $0.05 \mathrm{~mL}(=0.5 \mathrm{mg}$ Biliated bacillus of Calmette and Guerin). The dose of BCG (107 CFU / rat) was selected according to the indications provided by Moreau et al. (2008). The vaccine was administered intradermally (ID) in a dose of $0.02 \mathrm{ml} / \mathrm{rat}$, the first dose injected on the 9th day and the second on the 27th day of the experiment.

The control rats received an injection of $0.02 \mathrm{ml} /$ rat of $0.9 \%$ saline solution.

\section{Blood samples and hormonal assays}

The samples were taken on D0 in the basal state and at the end of the experimental period on the 32nd day. The blood samples are taken by retro orbital puncture in the morning between (8h-10h). Blood was collected in EDTA tubes for determination of Blood Count Formula and in HEPARINES tubes for analysis of plasma testosterone and adrenocorticotropic hormone.

\section{Hormonal dosage of plasma testosterone}

Blood samples are collected in heparin tubes and then centrifuged at $5000 \mathrm{rpm}$. Testosterone is measured by the conventional ELISA kit BIOTECH method. The measurement is made using a TECAN Magellan ELISA reader equipped with computer software which automatically calculates the standard range and gives us directly the value of the desired testosterone. This test was carried out by the Biomerieux MINI VIDAS device (100 $\mu$ l plasma serum). At the level of (laboratory of medical analyzes dr. benmahmoud sihem ép. fridja. z.h .u.n.n ${ }^{\circ} 5$ el bouni annaba .

\section{Hormonal dosage of ACTH}

Blood samples are collected in tubes (EDTA-plasma) after sacrifice or decapitation. This assay is carried out by the cobas e411 device, at the level (laboratory of medical analyzes dr. benmahmoud sihem ép. fridja. z.h .u.n. ${ }^{\circ} 5$ El Bouni Annaba .

\section{Statistical analysis}

The results are represented as an average \pm standard deviation (s). Statistical analysis was performed using 
Graph PadPrism 7 graphical statistical software where we requested the generalized linear model to make an overall statistical comparison of the sample means. This program automatically gives us the result of the comparison test of means with respect to the control average (One-way ANOVA test).

\section{Results}

Figure 1.Testosterone plasma level following cyclosporine injection and after noise application with combination of cyclosporine treatment in adult male Wistar rats.

Following one-way analysis of variance, the post-test Newman-Keuls for multiple comparisons showed a decreased significant difference in testosterone level after cyclosporine injection $(0,325)$ when compared with control group $(3,67),\left({ }^{*} \mathrm{P}=0,0366\right)$, where a highly significant decrease in testosterone mean level was observed in animals underwent cyclosporine injection with noise application $(0,3975)$ in comparison with control male rats $(3,67),\left({ }^{* *} \mathrm{P}<0.01\right)$. Newman-Keuls post-test, revealed a decreased significant difference in testosterone level after cyclosporin injection $(0,325),\left({ }^{*} \mathrm{P}=0,0186\right)$ and in male rats underwent cyclosporine injection with noise application 0,3975 when compared with basal group $(3,88),\left({ }^{*} \mathrm{P}=0,0211\right)$.

Figure 2. Adrenocorticotropic hormone plasma level following cyclosporine injection and after noise application with combination of cyclosporine treatment in adult male Wistar rats, $\left({ }^{*} \mathrm{P}<0.05,{ }^{* *} \mathrm{P}<0.01\right)$

Following one-way analysis of variance, the post-test Tukey's test for multiple comparisons showed a decreased significant difference in Adrenocorticotropic hormone plasma level after cyclosporine injection $(261,8)$ when compared with control group $(41,88),\left({ }^{*} \mathrm{P}=0,0224\right)$, where a highly significant decrease in Adrenocorticotropic plasma mean level $(261,8)$ was reached in comparison with basal male rats $(31,86)$, ${ }^{* *} \mathrm{P}$ $=0,0018)$. However, male rats underwent cyclosporine injection with noise application $(204,5)$ showed a statistical significant difference when compared with basal group $(31,86),\left({ }^{*} \mathrm{P}=0,0211\right)$.

Figure 3. Bodyweight variation at different days of measurement $(* * * \mathrm{p}<0.001)$

The ANOVA1 indicates statistically significant differences in body weight (grams) in the treated versus control groups (Control vs CsA: $\mathrm{q}=6.096,{ }^{* * *} \mathrm{P}<0.001$; Control vs CsA+Noise: $\mathrm{q}=6.784,{ }^{* * *} \mathrm{P}<0.001$ ).

\section{Discussion}

Effects of cyclosporine injection and cyclosporine associated with noise application in two groups of animals treated with a Biliated bacillus of Calmette and Guerin, were evaluated by measuring testosterone and adrenocorticotropic hormones plasma level. It has been reported that cyclosporine treatment of transplant patients can lead to abnormal gonadal function (Handelsman et al., 1984). This dysfunction of the gonadal function can be explained by the direct effect of cyclosporine on the testes or indirect mediated by other factors, even other pathways (oxidative stress factors, neuroendocrine factors, neuroimmune factors, etc.). Early work on the neuroendocrine effects of cyclosporine has suggested that this immunosuppressant may influence adrenal cortex function by increasing the secretion of pituitary corticotropic hormone (White et al., 1982). This is what we obtained from our results, there was a statistically insignificant increase in the level of adrenocorticotropic hormone in the treated animals. However, other reports have indicated that cyclosporine rather exerts an inhibitory effect on the hypothalamic-pituitary axis(Sikka et al., 1988), and can slow the growth and activity of adrenocortical steroid cells in the rat (Rebuffat et al., 1989) . In male rats, cyclosporine could inhibit or stimulate plasma lutenizing hormone level, depending on peripheral testosterone level(Sikka et al., 1988). Cyclosoprine administration increases serum prolactin level, selectively inhibits prolactin receptors and induces mild hyperglycemia independent of insulin levels (Kahan et al., 1989). The immune and endocrine systems are linked by a network of two-way interactions that, on the one hand, allow hormones to modulate immunological functions and, on the other, allow the immune system to produce neuroendocrine effects. (Thomas-Vaslin et al., 2017) . Hormones such as glucocorticoids, corticotropin, growth hormone, prolactin and somatostatin, are modulators of the immune and inflammatory responses while cytokines modulate the function of the corticotropic, gonadotropic, thyreotropic and somatotrope. These immuno-neuroendocrine interactions are therefore involved in many physiological situations (Srlf et al., 2014). They play a role in many physiological and pathophysiological 
situations. They thus play a role in hypogonadism, hypothyroidism and growth arrest that occur during infectious or inflammatory diseases. As for interactions with the hypothalamic-hypo-physo-adrenal axis, they allow the inflammatory response to be controlled in order to prevent an exaggeration of this response from endangering the integrity of the organism(Gaillard et al., 2003). Furthermore, it has been shown, in the gonadectomized rat that testosterone has a non-rapid genomic effect compared to estradiol which seems to have a rapid non-genomic effect. In another study, it was reported that both testosterone and estradiol exhibit anxiolytic and antidepressant-like effects in male gonadectomized rats, while similarly regulating the critical mediators of these behaviors, suggesting underlying mechanisms common(Khera et al., 2016) . Several hypotheses can be put forward to explain the choice of Biliated bacillus of Calmette and Guerin in our study. Biliated bacillus of Calmette and Guerin inoculation has been shown to cause persistent activation of Indole-amine-pyrrole 2,3-dioxygenase, the enzyme responsible for the breakdown of tryptophan, at the peripheral and brain levels in rats up to at three weeks, resulting in a decrease in tryptophan and therefore serotonin levels(Moreau et al., 2005) . This activation is mainly mediated by gamma interferon (Fujigaki et al., 2006) . Cytokines can have an indirect effect by inducing the secretion of the hypothalamic corticotropin exiting the hormone, which plays an important trophic role in adrenal activity. They have shown that interleukins IL-2 and IL-6 induce in vitro proliferation of anterior pituitary cells, as well as secretion of growth hormone, prolactin and adrenocorticotropic hormone. The reduction in thymic function can increase the synthesis of IL-1(Song et al., 2002). In addition, IL1 induces a change in certain neurotransmitter systems similar to those observed in thymectomized rats (Song et al., 2002). In addition, sex steroids are known for their suppressive effects on immune functions(Grossman et al., 1985) . Various steroids have also been reported to suppress in vitro proliferation of spleen cells in response to mitogens (Hirokawa et al., 1979). The physiological thymic breakthrough that begins around puberty can be attributed to the high levels of sex steroids. However, this thymic involution is not an irreversible phenomenon. In mice and rats, thymus atrophy at any age can be reversed, and the thymus can regain its size after gonadectomy (testicular or ovarian) (Utsuyama et al., 1995). Exposure of rats to uncontrollable painful electric shock (Dantzer et al., 2003) 24 hours after injection of syngeneic tumor cells leads to faster tumor development and higher mortality than in rats exposed to electric shock controllable, animals of the latter group do not differ from control animals not exposed to electric shock. The probability of rejection of a non-syngeneic tumor is also modified since it is lower in rats exposed to uncontrollable electrical shock 24 hours after implantation of the tumor than in rats exposed to controllable electrical shock and in control rats. (Dalle et al., 2007)Behavioral control attenuates the decrease in the cytotoxic activity of natural killer cells induced by inevitable electric shocks. In this context, we exposed male rats to noise (Schafer and Gleize 1979) (car horn) for eight days successively (for two minutes each day), and placed in a device. We have observed that the animals exhibit behavior reactive to the sound noise which fades within two days. (The rat turns on itself during one minute probably seeking the source of the noise, at the end of three days the rats remain frozen in reaction to the noise during the two minutes). As such noise is considered a factor of environmental stress, causing a wide range of health effects such as the acoustic, cardiovascular, nervous and endocrine systems. A study shows that structural changes observed at the apical pole of the ciliated cells of the inner ear (Surel et al., 2016) (ciliary fusion and cuticular plate anomaly) (El-Amraoui et al., 2005)therefore attest to the existence of a metabolic overload hair cells over-activated by moderate but long-term sound exposures. In addition, studies carried out on mice and guinea pigs have also shown neuronal degeneration in the absence of loss of hair cells. This suggests that the phenomenon is likely to be common to all mammals and therefore to humans undergoing repeated exposure to moderate noise. on the other hand, the results of Zheng (1997) have shown that intense exposure to noise is accompanied by a change in the weight of the adrenal gland in rats (Kapanadze, Abzianidze et al., 2009). The more or less intense vibrations, as a main element of noise, are thus perceived By the whole person. Indeed, these induce significant morphological or even pathological changes in the adrenal glands. The variation in noise exposure level in rats indicates that each area of the adrenal cortex shows a differential reaction to noise stress. These glandular changes may be due to an excessive release of catecholamines and corticosterone. The noise induces both a morphological alteration of the adrenal glands and of the myocardium. Therefore, long-term noise exposure can have a detrimental effect on health. Studies (Gunther et al., 1981) in rats have shown that chronic 
noise pollution alone or in combination with other stressors (eg, a magnesium-deficient diet) can lead to accelerated aging of the linked heart an increase in the secretion of stress hormones as well as an increased activity of the antioxidant mechanism in the heart(Gannouni et al., 2013). This trend was found in our study, where we recorded an increase in the ACTH level in the two groups of male wistar rats (Cyclosporine alone and cyclosporine + Noise).

\section{Conclusion and perspectives}

At the end of our study, we highlighted the immuno-neuroendocrine interaction. This study allowed us to assess and explore the effect of cyclosporine administration (Cyclosporine) alone and the effect of cyclosporine associated with noise in animals treated with Biliated bacillus of Calmette and Guerin. The results obtained show that cyclosporine alone and cyclosporine associated with noise have the same effects in the male rat. This implies that the sound processing mimics the effect of cyclosporine. The results of this study underline the value of using sound therapy as a treatment to improve the lives of patients and reduce their suffering, also to avoid the use of certain drugs. In this context, it would be interesting to carry out research perspectives in this field and this by carrying out the following experiments:

-Test the combined effect (Cyclosporine + Noise) on females to compare them to the male through hormonal variations

-Test the combined effect (Cyclosporine + Noise) on humans (healthy, sick)

-Test the effect of administering cyclosporine to different routes by applying different doses.

Funding details: There were no funding sources for this study

Declaration of interest statement: The authors report no conflict of interest.

Ethics of experimentation: All applicable international, national, and/or institutional guidelines for the care and use of animals (The Ethical Committee grants positive opinion for use of Wistar rats for the project supported by the ministry of higher Education and Scientific Research, which is consistent with the principles of Animal Health: NIH Publication No. 85-23, revised 1985. D01N01UN230120150001) were followed.

\section{References}

Ader, R., et al , Annual Review of Pharmacology and Toxicology, "Interactions between the brain and the immune system.", 1990, 30(1): 561-602.

Bataille, G., et al , Journees de la Recherche Porcine en France, "Consequences comportementales, zootechniques et physiologiques de l'epointage des dents chez le porcelet.", 2002, 34: 203-210.

Billy, C. and C. Perronne, EMC-Maladies infectieuses, "Aspects cliniques et therapeutiques de la tuberculose chez l'enfant et l'adulte.", 2004, 1(2): 81-98.

Bockaert, J. and C. Becamel, medecine/sciences, "Controle de l'anxiete par les recepteurs de la serotonine 5-HT2c de la strie terminale-Une explication des effets anxiogenes des inhibiteurs selectifs de la recapture de la serotonine (ISRS)?", 2017, 33(1): 87-89.

Borel. JF., Feurer. C., Gubler. HU., and Stahelin H, Agents and Actions, Biological effects of CsA: a new antilymphocytic agent, 1976, 6:468-75

Borlongan. CV., Stahl. CE., Cameron. D., Saporta. S., Freeman. TB., Cahill. D.W., and Sanberg, P.R , Neurol. Res, CNS immunological modulation of neural graft rejection and survival, 1996, 18: 297-304.

Borlongan. CV., Fujisaki. T., and Watanabe. S, Neuro Report, Chronic administration of Cyclosporine-A does not impair memory retention in rats, 1997, 8 :673-676. 
Borlongan, CV., Stahl, CE., Fujisaki, T., Sanberg, PR. and Watanabe, S , Cell Transplant, Cyclosporine A- induced hyperactivity in rats: is it mediated by immunosuppression,neurotrophism, or both?, 1999, 8: 153-159

Borlongan. CV., Stahl. C E., Keep. MF., Elmer. E., and Watanabe. S , Neuroscience Letters, Cyclosporine-A enhances choline acetyltransferase immunoreactivity in the septal region ofadult rats, 2000, $279: 73-76$.

Cohen, D. J., et al, Annals of internal medicine, "Cyclosporine: a new immunosuppressive agent for organ transplantation.", 1984, (5): 667-682.

Dantzer, R. and E. E. Wollman, Journal de la Societe de Biologie, "Les inter-relations entre le systeme nerveux et le systeme immunitaire.", 2003, 197(2): 81-88.

De Groen. PC., Aksamit. AJ., Rakela. J., Forbes. GS. and Krom RA , NEngl J Med, Centralnervous system toxicity after liver transplantation. The role of cyclosporine and cholesterol, 1987, $317: 861-866$.

Elder, A., et al , Environmental health perspectives, Translocation of inhaled ultrafine manganese oxide particles to the central nervous system.", 2006, 114(8): 1172.18:5151-5159.

Fauve, R , Immunology, "Inflammation and natural immunity.", 1980, 80: 737-756.

Friberg, H., Ferrand-Drake, M., Bengtsson, F., Halestrap, AP. and Wieloch, T. 1998.

Fujigaki, H., et al , Journal of Biochemistry, "The signal transducer and activator of transcription $1 \alpha$ and interferon regulatory factor 1 are not essential for the induction of indoleamine 2, 3-dioxygenase by lipopolysaccharide: involvement of p38 mitogen-activated protein kinase and nuclear factor- $x \mathrm{~B}$ pathways, and synergistic effect of several proinflammatory cytokines.", 2006, 139(4): 655-662.

Gaillard, R.-C , Journal de la Société de Biologie, "Les interactions immuno-neuroendocriniennes et leurs implications cliniques.", 2003, 197(2): 89-95.

Gannouni, N., et al , Evaluation des atteintes cochléaires et des troubles extra-auditifs chez des rats mâles exposés d'une façon répétitive au bruit assessment of cochlear effects and extra-auditory disorders in male rats exposed repetitively to low noise.", 2013.

Genard, R, Université Paris-Saclay, Rôle du facteur de transcription Nrf2 dans l'immunomodulation induit par les adjuvants vaccinaux, 2015.

Gerbase, M , Rev Med Suisse, "Immunostimulants chez les syndrômes obstructifs chroniques.", 2000, 4: 20603.

Grastyan, E., et al, "The hippocampal electrical correlates of the homeostatic, 1966.

Grossman C.J, Science, Interaction between the gonadal steroids and the immune system, 1985, 227:255261.

Günther, T , Electroencephalography and clinical neurophysiology, "Biochemistry and pathobiochemistry of magnesium, 1981, 9(3): 167-181.

Handelsman. DJ., McDowell. IFW., Caterson. ID., Tiller. D., Hall, BM. and Turtel. JR , Clin. Nephrol, Testicular function after renal transplantation: comparison of cyclosporine a with azathioprine and prednisone combination regimes, 1984, $22: 144-148$.

Hill, Q. A., et al , British journal of haematology, "Guidelines on the management of drug-induced immune and secondary autoimmune, haemolytic anaemia.", 2017, 177(2): 208-220. 
Hirokawa K., Okayasu I., Hatakeyama Hatakeyama S, Acta Pathol Japon, Effect of pregnancy and its related hormones on the in vitro proliferation of spleen cells, 1979, 29:837-844.

Jarius, S. and B. Wildemann, European neurology, "Pavlov's Reflex before Pavlov: Early Accounts from the English, French and German Classic Literature.", 2017, 77(5-6): 322-326.

Kahan, B. D , "Cyclosporine.", New England Journal of Medicine, 1989, 321(25): 1725-1738.

Kalantaridou, S., et al,Journal of reproductive immunology, "Stress and the female reproductive system.", 2004, 62(1-2): 61-68.

Kamilia, G., et al , "Administering ketoconazole $(25 \mathrm{mg} / \mathrm{kg})$ for 14 days in male wistar rat provokes testicular damage accompanied by changes in testosterone levels and immune function, 2015.

Kapanadze, N., et al, Georgian medical news, "Morphological structure of suprarenal glands in experimental vibration-induced pathology.", 2009, 166): 78-80.

Karten, M. J. and J. E. Rivier, Endocrine Reviews, "Gonadotropin-releasing hormone analog design. Structure-function studies toward the development of agonists and antagonists: rationale and perspective.", 1986, 7 (1): 44-66.

Kelley, K. W., et al , Brain, behavior, and immunity, "Cytokine-induced sickness behavior.", 2003, 17(1): 112-118.

Khera, M., et al , The journal of sexual medicine, "Diagnosis and treatment of testosterone deficiency: recommendations from the Fourth International Consultation for Sexual Medicine (ICSM 2015).", 2016, 13 (12): 1787-1804.

MacAdams. MR., White. RH. and Chipps BE, Ann. Intern. Med, Reduction in serum testosterone levels during chronic glucocorticoid therapy, 1986, 140:648-651.

Mihatsch MJ., and Wolff, K , Br, J . Dermatol, Consensus conference in cyclosporine A for psoriasis, 1992, 126:621-623.

Moreau M., Lestage J., Verrier D., Mormede C., Kelley K.W., Dantzer R. and Castanon N , Journal of Infectious Diseases, Bacille Calmette-Guerin inoculation induces chronic activation of peripheral and brain indoleamine 2,3-dioxygenase in mice, 2005, 192(3): 537-544.

Nussenblatt RB, Jpn J Ophthalmol, Basic and clinical immunology in uveitis, 1987, 31(3):368-74.

Rabin. D., Gold. P.W., and Margioris. A, Plenum Press New York, Stress and reproduction: Interactions between the stress and reproductive axis. In: Chrousos GP, Loriaux DL, Gold PW (eds) Mechanisms of physical and emotional stress, 1988, 377-390.

Rebuffat. P., Cavallini. L., Belloni. AS., Mazzocchi. G., Coi. A., de Tos. GP., and Nussdorfer. GG , A morphometric study on the reversal of ACTH-induced hypertrophy of rat adrenocortical cells after cessation of treatment J Submicrosc Cytol Pathol, 1989, 21: 73-81.

Rivier. C., And Rivest S , Biol. Reprod, Effect of stress on the activity of the hypothalamic-pituitarygonadal axis: Peripheral and central mechanisms, 1991, 45:523-532.

Rivier. C., Rivier. J. and Vale W , Reanimation,Stress-induced inhibition of reproductive function: Role of endogenous corticotropin releasing factor. Science, 1986, 231:607-609.

Schafer, R. M. and S. Gleize, Lattes Paris, Le paysage sonore, J.-C, 1979.

SIKKA, S. C., et al , Endocrinology, "Effects of cyclosporine on the hypothalamic-pituitary-gonadal axis in the male rat: mechanism of action.", 1988, 123(2): 1069-1074.

Sikka, S. C., et al , Transplantation, "Reversibility of cyclosporine-induced hypoandrogenism in rats", 1988, 45(4): 784-787. 
Skarbek, C , Universite Paris-Saclay, Synthese et evaluation pharmacologique d'analogues preactives de l'ifosfamide: prodrogues et nanoparticules a visee antitumorale, 2017.

SRLF , Reanimation, "Immunite/dysfonction cellulaire.", 2014, 24: S47-S50.

Song , C , BrainBehavior and Immunity, The effect of thymectomy and IL-1 on memory: Implications for the relationship between immunity and depression, 2002, 16: 557-568.

Stephan, BC., Douglas, EL., and Diane, HR , Biophys. Res. Commun. Biochem, Rapid elevation of serum prolactin concentrations by cyclosporine, a novel immunosuppressive drug, 1984, 12: 614-618.

Stora, J.-B , "Chapitre IV. Mecanismes neurobiologiques et maladies du stress." Que sais-je?, 2016, 9: 83-103.

Surel, C, Universite Montpellier, Les mecanismes de la neuropathie auditive AUNA1, 2016.

Utsuyama, M., et al , Mechanisms of ageing and development, "Differential effects of gonadectomy on thymic stromal cells in promoting T cell differentiation in mice.", 1995, 81 (2-3): 107-117.

1. Author name(s), journal or book title, article or chapter title, year of publication, volume and issue (where appropriate) and page numbers.

2.

Figures

Fig. 1. Testosterone plasma level following cyclosporine injection and after noise application with combination of cyclosporine treatment in adult male Wistar rats, $\left({ }^{*} \mathrm{P}<0.05,{ }^{*} \mathrm{P}<0.01\right)$

Fig. 2. Adrenocorticotropic hormone plasma level following cyclosporine injection and after noise application with combination of cyclosporine treatment in adult male Wistar rats, $\left({ }^{*} \mathrm{P}<0.05,{ }^{*} \mathrm{P}<0.01\right)$

Fig. 3. Bodyweight variation at different days of measurement $(* * * \mathrm{p}<0.001)$

Figure captions

1. Testosterone plasma level following cyclosporine injection and after noise application with combination of cyclosporine treatment in adult male Wistar rats, $\left({ }^{*} \mathrm{P}<0.05,{ }^{* *} \mathrm{P}<0.01\right)$

2. Adrenocorticotropic hormone plasma level following cyclosporine injection and after noise application with combination of cyclosporine treatment in adult male Wistar rats, $\left({ }^{*} \mathrm{P}<0.05,{ }^{* *} \mathrm{P}<0.01\right)$

3. Bodyweight variation at different days of measurement $(* * * \mathrm{p}<0.001)$ 